\title{
FIGO IVB cervical cancer patients benefit from locoregional surgery: a retrospective study from the SEER database
}

\section{Yanhong Wang ( $\sim 992415639 @ q q . c o m$ )}

Second Affiliated Hospital of Fujian Medical University https://orcid.org/0000-0001-8152-0574

\section{Yi Ouyang}

Sun Yat-Sen University Cancer Center Department of Radiotherapy

Jingjing Su

Second Affiliated Hospital of Fujian Medical University

\section{Zhigang Bai}

Second Affiliated Hospital of Fujian Medical University

\section{Qunrong Cai}

Second Affiliated Hospital of Fujian Medical University

\section{Xinping Cao}

Sun Yat-Sen University Cancer Center Department of Radiotherapy

\section{Research article}

Keywords: Cervical cancer, Distant metastasis, Surgery, Prognosis, SEER.

Posted Date: November 20th, 2019

DOI: https://doi.org/10.21203/rs.2.17587/v1

License: () (1) This work is licensed under a Creative Commons Attribution 4.0 International License. Read Full License 


\section{Abstract}

Background: We aimed to analyze the clinical value of primary site surgery in improving survival of initial metastatic cervical cancer. Methods: A population-based retrospective study which analyzed clinical data extracted from the National Cancer Institute's Surveillance, Epidemiology, and End Results (SEER) database was conducted. Stratified analysis was employed to evaluate the effect of cervical surgery on cervical cancer specific survival (CCSS) and overall survival (OS). Then COX regression models were performed to adjust potential confounders, and assess the survival benefit of cervical surgery for patients with primary metastatic cervical cancer. Results: The median CCSS and OS in the surgery group were more than twice of that in the group without surgery. Primary site surgery conferred prognosis superiority for patients with metastases merely to lung, and other site or distant LN, but not multiple metastasis and bone, liver. Pelvic lymph node dissection conducted in combination with cervical surgery provided a survival advantage over hysterectomy. Moreover, an aggressive treatment that integrated locoregional surgery with radiotherapy or chemotherapy showed the better survival when compared to surgery alone. The survival advantage provided by primary site surgery was not influenced by the histological type, lymph node status. Finally, after adjusting confounders using COX regression, local cervical surgery reduced the cancer related and overall mortality rate by about $30 \%$. Conclusions: Surgical procedures could promote the survival of patients with primary metastatic cervical cancer, and should be considered as a therapeutic option for carefully chosen patients.

\section{Background}

Cervical cancer remains to be one of the most common tumors affecting women worldwide, ranking third for cancer incidence and fourth for mortality(1). Among patients with newly diagnosed cervical cancer, roughly $10 \%$ have distant metastasis at their initial diagnosis (2), and have a dismal prognosis, with poor median survival time usually less than 1 to 2 years $(3,4)$.

For International Federation of Gynecology and Obstetrics (FIGO) stage IVB cervical cancer, platinumbased chemotherapy, angiogenesis inhibitor bevacizumab and immunotherapy are the choice of treatment, and continuous to be considered palliative. Moreover, the studies of locoregional radiation therapy combined with system therapy for primary metastatic cervical cancer are emerging, and showed that locoregional radiotherapy would confer a substantial longer survival than system therapy alone(510). Nevertheless, the role of local surgery for these cervical cancer patients is still not established. In tradition, since FIGO IVB cervical cancer is considered incurable, surgical treatment is only recommend as a palliative treatment to relieve symptom such as pain, bleeding, and obstruction. Additionally, it has been reported that primary tumor mass inhibit remote metastasis by a circulating angiongenesis inhibitor, and once tumor removal, metastasis neovascularize and grow (11), but this viewpoint has not supported by clinical evidence. Growing studies have demonstrated prolonged survival of metastatic diseases when aggressive local surgeries were carried out (12-16). Sriram Venigalla and his colleagues proved that cervical cancer patients with disseminated disease would benefit from locally definitive treatment (concurrent chemotherapy or definitive surgery), and median OS time elevated 9.1 months. However, only 
$14 \%$ patients of definitive treatment group undergo definitive surgery, and the relative role of surgery on survival was not investigated (7).

To further examine the benefits of primary tumor resection for cervical cancer patients, we analyzed a large data from the National Cancer Institute's Surveillance, Epidemiology, and End Result (SEER) program database to conduct this population-based epidemiologic study. Sites of metastasis, histological type, surgical pattern, chemotherapy, radiotherapy, T stage and lymph node status were stratified in the analysis of OS and cervical cancer specific survival (CCSS). We hypothesized that FIGO IVB cervical cancer patients would benefit from locoregional surgery.

\section{Methods}

\section{Data Acquisition and Processing}

We identified cervical cancer cases from the SEER database using the official software SEERStat 8.3.6 on September 20, 2019. The demographic, clinicopathological and follow-up data of 23873 women with cervical cancer diagnosed from 2010 to 2016 was obtained. After screening the data based on the 7th AJCC staging system, 1483 IVB patients with at least one distant metastasis and definite information on surgery of primary site were included in this study.

Patients were classified based on whether they underwent locoregional surgery to remove the primary cervical tumor after the initial diagnosis. Age at diagnosis was categorized into three groups, younger than 40,41 to 60,61 to 80,81 and older. Based on race, patients were categorized as White, Black, and others. Histological type included squamous cell carcinoma (SCC), adenocarcinoma (ADC), and others. Based on 7th AJCC staging system, patients with N0 were allocated as lymph node negative, N1 were positive. According to surgery pattern, patients were assigned into the "Hysterectomy" and "Radical Hysterectomy" groups. Data on chemotherapy, radiation, distant surgery were extracted, but the exact regimens, cycles, dose, and site were not in the SEER database.

\section{Statistical analysis}

Chi-square tests or Wilcoxon signed-ranks test were used to compare the distribution difference of locoregional surgery and demographic characteristics. For CCSS, only death due to cervical cancer was considered as an event occurrence, while for OS, it was death due to any cause. Survival time was calculated using Kaplan-Meier methods, and compared by log-rank test. Univariate and multivariate Cox proportional hazards model was performed to evaluate the effects of demographic factors on CCSS and OS using the hazard ratio (HR) and $95 \%$ confidence intervals $(95 \% \mathrm{Cl})$. Variables that showed statistical significance in the univariate analysis or were considered as important for survival were then enrolled in multivariate analysis using a stepwise conditional method. Data analysis was performed using the software SPSS 22.0 and a 2 -sided $p$ value of less than 0.05 was considered to be statistically significant. 


\section{Results}

Demographic characteristics of the patients

Among 1483 patients with FIGO IVB cervical cancer diagnosed between 2010 and 2016, 336 (22.6\%) of them underwent surgery, while 1147 (77.3\%) did not. The clinicopathological characteristics of patients with metastatic cervical cancer are summarized in Table1. The median age for the non-surgery group was 56 years, while it was 52 years for surgery group, indicating that older patients were more prone to choose conservative treatment. Race did not appear to affect the decision to excision the primary site tumor. The tumors resected from patients who had cervix surgery were less likely to be squamous cell carcinoma, and more likely to be T1 and T2 (both $\mathrm{p}<0.0001$ ). Compared to non-surgery group, the surgery group had more patients with negative lymph node $(27.4 \%$ vs. $36.6 \%, p=0.001)$. Less patients with multiple metastatic sites would opt for surgery, and more patients in the surgery group had other site or distant LN affected. There were more patients in surgery group deciding to remove distant metastases and received systemic chemotherapy. More patients received radiotherapy if they did not undergo cervix surgery.

Analysis of CCSS and OS

To analyze the effects of local surgery on CCSS and OS in patients with metastatic cervical cancer, log-rank test were performed between surgery and non-surgery groups. As illustrated in Fig. 1, locoregional surgical treatment conferred a prominent survival advantage. The median CCSS and OS were 37 and 28 months for the surgery group, 13 and 11 months for the non-surgery group (Fig. $1 \mathrm{~A}$ and Additional file 1).

In order to eliminate the bias of site-specific metastasis on survival analysis, data were stratified based on the distant organs involved (multiple site, bone, liver, brain and lung, other site or distant LN), and CCSS was evaluated based on whether or not surgery of the primary tumor was performed in mentioned-above patient groups. Owing to the limited number of patients with isolated brain metastasis, they were excluded from this evaluation. Patients with isolated lung and other site or distant LN involved benefitted from primary site surgery ( $p=0.03$ for lung only; $p<0.0001$ for other site or distant LN) (Fig. $1 E, F)$. Nevertheless, the locoregional treatment could not prolong the survival of patients with bone, liver and multiple sites metastasis. Furthermore, the aggregate effects of surgical resection of primary and metastatic lesions on survival were also evaluated. It showed that survival was better for patients who underwent resection for distant organs, and significantly better for patients whose primary cervical tumors were resected (Fig. 2A).

Local surgeries were categorized into the "Hysterectomy" group (without regional lymph node dissection) and "Radical hysterectomy" group (with regional lymph node dissection). In all cases, pelvic lymph node dissection provided additional survival advantage to the cervical cancer patients (Fig. 2B). 
The effects of chemotherapy and/or radiotherapy together with local surgery on survival in patients with metastatic cervical cancer were next analyzed (Fig. 2C-F). Prognosis was better for patients who underwent locoregional resection combined with chemotherapy or radiation compared with those received surgery/chemotherapy/radiation alone. Chemotherapy conferred similar OS and CSSS to locoregional surgery, while radiation provided worse survival compared to surgical treatment.

Data stratified on the basis of histology, and lymph node status showed that they did not affect the survival advantage provided by locoregional surgery. More advanced AJCC T stage, less advantage gained from primary site surgery. Eventually, survival benefit disappeared for T4 tumor.

Univariate and multivariate analysis

\section{Univariate and multivariate analysis were then performed to evaluate the effects of the} clinicopathological factors on CSSS and OS (Table 2). As illustrated in Table 2, patients with age between 41 and $\mathbf{8 0}$ were at a lower risk of cancer-related death compared to those younger than 40 . Compared to white race, black women were at a higher risk of mortality. Tumors with higher AJCC T stage had a poor prognosis. When the lymph node was affected, CCSS was worse than in cases with negative nodes. For treatment, regional LN surgery, chemotherapy and radiation prolonged cancer-related and overall survival for cervical cancer with primary metastasis, while surgery of distant metastasis did not show any significance in CSSS and OS. Except for only brain metastasis, women with isolated organ metastases had better OS and CSSS compared to those with multiple distant organ involved.

\section{Local cervix surgery reduced the cancer-associated and overall mortality rate by about $33 \%$ and $31 \%$, which demonstrated that regional resection of primary tumor is an independent prognosis factor and is of vital significance in prolonging the survival of cervical cancer patients with primary metastasis.}

\section{Discussion}

Usually, the treatment for FIGO IVB cervical cancer has been and continues to be considered palliative. And systemic therapies including platinum-based chemotherapy, target therapy and immunotherapy have been mainstay of care for patients with metastatic cervical cancer (3). In recent years, the efficacy of locoregional management on improving survival in $\mathrm{M} 1$ cervical cancer has been highlighted in multiple retrospective studies. A retrospective study by Tomoyuki et al. indicates that treatment with definitive radiotherapy was associated with improved survival compared with chemotherapy or palliative care alone (2). Another study by Sriram et al., with a large sample size of 2838 indentified from National Cancer Database, revealed that patients who underwent definitive local therapy (either concurrent chemoradiation or surgery) had a $43 \%$ reduced mortality risk compared to those who received conservative therapy (systemic therapy with or without palliative radiation)(7). Similar conclusion was drawn by a Chinese study, which illustrated that chemotherapy combined with definitive pelvic radiotherapy would significantly prolong the OS by 7.3 months when compared to chemotherapy alone or with palliative pelvic radiotherapy(5). Another two studies which enrolled patients from SEER or National 
Cancer Database, also demonstrated that radiotherapy would decrease $28-31 \%$ mortality risk for primary metastatic cervical cancer $(6,8)$.

However, in the literature, the resection of primary tumor for patients with FIGO IVB cervical cancer is poorly documented. In this study, based on SEER database, we found that excision of the primary cervical tumor, in general, could bring a survival benefit for metastatic cervical cancer, reducing the risk of mortality by about $30 \%$. Several plausible reasons might help to explain why pelvic surgery would contribute to improving the prognosis in patients with metastatic breast cancer. Firstly, local surgery could mitigate symptoms such as pain, bleeding and infection, which poses a risk of morbidity and mortality that is independent of distant disease(17). However, systemic therapy alone is often insufficient to address such consequences of local progression. Secondly, control of the primary site may reduce the risk of further seeding of malignant cells and thereby reduce the risk of development new distant dissemination (7). Thirdly, surgical removal of the primary tumor decreases the production of immunosuppressive cytokines thereby, reversing tumor-induced immunosuppression and allow the host immune system to regain immunologic control of systemic disease burden(18). Fourthly, surgery removes the necrotic centric areas that are hypoxic and resistant to chemotherapy and radiotherapy, therefore, increasing the efficacy of adjuvant treatment and improving prognosis (19-21).

Although local surgery poses a benefit of survival, it is worth underlining that such therapy is likely not suitable for all patients with metastatic disease. The stratified analysis in this study demonstrated that hysterectomy resulted in survival improvement in cervical cancer patients with metastasis to lung alone and other site or distant LN alone. In line with previous studies, patients with metastasis to pulmonary or distant lymph node alone has remarkably good prognosis and gains survival benefit from aggressive therapy $(22,23)$. Hence, local surgery may be an appropriate treatment option for a carefully selected group of patients with good performance status, symptomatic primary disease and a low metastatic disease burden. Multiple studies have emphasized the risk of a positive surgical margin on prognosis (24, 25). Unfortunately, we could not obtain data on surgical margin status from the SEER database. We found that the advantage of surgery did not showed in patients with T4 stage (invasion into neighboring structures such as bladder and rectum), and there were prominent survival differences between women who had hysterectomy and those had a radical hysterectomy. It's well known that a clear surgical margin is difficult to achieve in patients with tumors progression to neighboring organs. Pelvic lymph node dissection would also contribute to lower the tumor burden, and stop cancer cell spreading to distant organs through lymphatic pathway. Consequently, we preferentially hold the opinion that definitive surgery to remove primary tumor clearly vitally improves the therapeutic efficacy by lowering the tumor burden.

As for treatment, radiotherapy and chemotherapy were also independent prognostic factors for FIGO IVB cervical cancer. Moreover, an aggressive treatment approach that combines chemotherapy or radiation with surgical management of primary tumors may lead to improved survival compared with abovementioned treatments alone, and the survival advantage of combined treatment over palliative treatment is even greater than that of single treatment add together. Therefore, local surgical combined 
with systemic therapy is recommended for primary metastatic cervical cancer with good performance. Regrettably, SEER database does not include information on chemotherapy regimen and cycles or radiation field size and dose, which would have allowed us to analyze survival based on chemotherapy modalities as well as the extent of radiotherapy administered.

There were several inevitable limitations in the present study. First, there is an inherent patient selection bias as it is a population-based retrospective study. Second, details of the systemic therapy, radiotherapy, performance status, and surgical margin were lacking, which could also affect prognosis. Third, the detail on how metastasis diagnosed was unavailable, which may affect the result to some extent. Consequently, these results should be prudently interpreted with these limitations in mind.

\section{Conclusions}

Surgical procedures in combination with lymph node resection, chemotherapy and radiotherapy have some positive effects on patients with metastasis to lung alone and other site or distant LN alone, a small primary tumor except for T4. Further well-designed studies should be initialed to indentify advantage of surgery over palliative therapy; also the surgical margin, systemic treatment strategy, and diagnosis method of metastasis deserve more attention.

\section{Abbreviations}

AJCC: American Joint Committee on Cancer

ADC: adenocarcinoma

CCSS: Cervical cancer specific survival

FIGO: International Federation of Gynecology and Obstetrics

LN: Lymph Node

OS: Overall Survival

SCC: squamous cell carcinoma

SEER: Surveillance, Epidemiology, and End Results

\section{Declarations}

Ethics approval and consent to participate

The SEER database was publicly available and all the research data were de-indentified. So no ethic approval and informed consents were needed in this study.

Consent for publication 
Not applicable.

Availability of data and materials

The data were retrieved from publicly accessible database "Surveillance, Epidemiology, and End Results" (SEER), the website is "https://seer.cancer.gov/". The definite data used in this study is available from the corresponding author on reasonable request.

Competing interests

All authors declare that they have no conflict of interest.

Funding

This work was supported by Science and Technology Planning Project of Quanzhou Science and Technology Bureau grant number [2018N001S] and Fujian Provincial Health and Family planning Commission Research Talent training project grant number [2018-1-60].

Authors' contributions

CQR and CXP participated in the design of the study. WYH and CQR collected the data from SEER database. SJJ and CQR did the literature search. OYY and BZG play a chief role in statistical part. WYH and OYY drafted the main manuscript. All authors have read and approved the final manuscript.

Acknowledgements

Not applicable.

\section{References}

1. Siegel RL, Miller KD, Jemal A. Cancer Statistics, 2017. CA: a cancer journal for clinicians. 2017;67(1):7-30.

2. Sasano T, Mabuchi S, Kuroda H, Takahashi R, Kozasa K, Isohashi F, et al. Predictors of Survival in Patients With FIGO Stage IVB Cervical Cancer. International journal of gynecological cancer : official journal of the International Gynecological Cancer Society. 2016;26(3):528-33.

3. Cohen PA, Jhingran A, Oaknin A, Denny L. Cervical cancer. Lancet (London, England). 2019;393(10167):169-82.

4. Waggoner SE. Cervical cancer. Lancet (London, England). 2003;361(9376):2217-25.

5. Yin Z, Lou H, Tang H, Ni J, Zhou Q, Chen M. Efficacy of radical doses of pelvic radiotherapy for primary tumor treatment in patients with newly diagnosed organ metastatic cervical cancer. 2019;14(1):82.

6. Wang Y, Farmer M, Izaguirre EW, Schwartz DL, Somer B, Tillmanns T, et al. Association of Definitive Pelvic Radiation Therapy With Survival Among Patients With Newly Diagnosed Metastatic Cervical 
Cancer. JAMA oncology. 2018;4(9):1288-91.

7. Venigalla S, Guttmann DM, Horne ZD, Carmona R, Shabason JE, Beriwal S. Definitive local therapy is associated with improved overall survival in metastatic cervical cancer. Practical radiation oncology. 2018;8(6):e377-e85.

8. Huang K, Jia M, Li P, Han J, Zhang R, Li Q, et al. Radiotherapy Improves the Survival of Patients With Metastatic Cervical Cancer: A Propensity-Matched Analysis of SEER Database. International journal of gynecological cancer : official journal of the International Gynecological Cancer Society. 2018;28(7):1360-8.

9. Hata M, Koike I, Miyagi E, Asai-Sato M, Kaizu H, Mukai Y, et al. Radiation Therapy for Patients with Bone Metastasis from Uterine Cervical Cancer: Its Role and Optimal Radiation Regimen for Palliative Care. Anticancer research. 2018;38(2):1033-40.

10. Kim JY, Kim JY, Kim JH, Yoon MS, Kim J, Kim YS. Curative chemoradiotherapy in patients with stage IVB cervical cancer presenting with paraortic and left supraclavicular lymph node metastases. International journal of radiation oncology, biology, physics. 2012;84(3):741-7.

11. O'Reilly MS, Holmgren L, Shing Y, Chen C, Rosenthal RA, Moses M, et al. Angiostatin: a novel angiogenesis inhibitor that mediates the suppression of metastases by a Lewis lung carcinoma. Cell. 1994;79(2):315-28.

12. Shi X, Huang NS, Shi RL, Wei WJ, Wang YL, Ji QH. Prognostic value of primary tumor surgery in minor salivary-gland carcinoma patients with distant metastases at diagnosis: first evidence from a SEER-based study. Cancer Management \& Research. 2018;10:2163-72.

13. Luo D, Liu Q, Yu W, Ma Y, Zhu J, Lian P, et al. Prognostic value of distant metastasis sites and surgery in stage IV colorectal cancer: a population-based study. International journal of colorectal disease. 2018;33(9):1-9.

14. Luo D, Liu Q, Yu W, Ma Y, Zhu J, Lian P, et al. Prognostic value of distant metastasis sites and surgery in stage IV colorectal cancer: a population-based study. 2018;33(9):1241-9.

15. Chen JQ, Kong YY, Weng SS, Dong CX, Zhu LZ, Yang ZR, et al. Outcomes of surgery for gastric cancer with distant metastases: a retrospective study from the SEER database. Oncotarget. 2017;8(3):434251.

16. Rafii A, Deval B, Geay JF, Chopin N, Paoletti X, Paraiso D, et al. Treatment of FIGO stage IV ovarian carcinoma: results of primary surgery or interval surgery after neoadjuvant chemotherapy: a retrospective study. International journal of gynecological cancer : official journal of the International Gynecological Cancer Society. 2007;17(4):777-83.

17. Ramondetta L. What is the appropriate approach to treating women with incurable cervical cancer? Journal of the National Comprehensive Cancer Network : JNCCN. 2013;11(3):348-55.

18. Danna EA, Sinha P, Gilbert M, Clements VK, Pulaski BA, Ostrand-Rosenberg S. Surgical removal of primary tumor reverses tumor-induced immunosuppression despite the presence of metastatic disease. Cancer research. 2004;64(6):2205-11. 
19. Lara PC, Lloret M, Clavo B, Apolinario RM, Henriquez-Hernandez LA, Bordon E, et al. Severe hypoxia induces chemo-resistance in clinical cervical tumors through MVP over-expression. Radiation oncology (London, England). 2009;4:29.

20. Markowska J, Grabowski JP, Tomaszewska K, Kojs Z, Pudelek J, Skrzypczak M, et al. Significance of hypoxia in uterine cervical cancer. Multicentre study. European journal of gynaecological oncology. 2007;28(5):386-8.

21. Rofstad EK, Sundfor K, Lyng H, Trope CG. Hypoxia-induced treatment failure in advanced squamous cell carcinoma of the uterine cervix is primarily due to hypoxia-induced radiation resistance rather than hypoxia-induced metastasis. British journal of cancer. 2000;83(3):354-9.

22. Kim TH, Kim MH, Kim BJ, Park SI, Ryu SY, Cho CK. Prognostic Importance of the Site of Recurrence in Patients With Metastatic Recurrent Cervical Cancer. International journal of radiation oncology, biology, physics. 2017;98(5):1124-31.

23. Jalloul RJ, Sharma S, Tung CS, O'Donnell B, Ludwig M. Pattern of Care, Health Care Disparities, and Their Impact on Survival Outcomes in Stage IVB Cervical Cancer: A Nationwide Retrospective Cohort Study. International journal of gynecological cancer : official journal of the International Gynecological Cancer Society. 2018;28(5):1003-12.

24. McCann GA, Taege SK, Boutsicaris CE, Phillips GS, Eisenhauer EL, Fowler JM, et al. The impact of close surgical margins after radical hysterectomy for early-stage cervical cancer. Gynecologic oncology. 2013;128(1):44-8.

25. Khanna N, Rauh LA, Lachiewicz MP, Horowitz IR. Margins for cervical and vulvar cancer. Journal of surgical oncology. 2016;113(3):304-9.

\section{Tables}


Table 1. Clinicopathological characteristics of women diagnosed with primary metastatic cervical cancer included in SEER database.

\begin{tabular}{|c|c|c|c|}
\hline & No surgery & Surgery & $\mathrm{p}$ \\
\hline Age & $56(47-65)$ & $52(43-62)$ & 0.000 \\
\hline Race & & & 0.169 \\
\hline White & $849(74.0)$ & $256(76.2)$ & \\
\hline Black & 192(16.7) & $43(12.8)$ & \\
\hline Others & $106(9.2)$ & $37(11.0)$ & \\
\hline Grade & & & 0.598 \\
\hline I & $49(4.3)$ & $17(5.1)$ & \\
\hline II & $371(32.3)$ & $117(34.8)$ & \\
\hline III & $661(57.6)$ & $180(53.6)$ & \\
\hline IV & $66(5.8)$ & $22(6.5)$ & \\
\hline Histological type & & & 0.000 \\
\hline SCC & $792(69.0)$ & $161(47.9)$ & \\
\hline $\mathrm{ADC}$ & $234(20.4)$ & $116(34.5)$ & \\
\hline Others & $121(10.5)$ & $59(17.6)$ & \\
\hline T stage & & & 0.000 \\
\hline $\mathrm{T} 1$ & $172(15.0)$ & 108(32.1) & \\
\hline $\mathrm{T} 2$ & $260(22.7)$ & $106(31.5)$ & \\
\hline $\mathrm{T} 3$ & $525(45.8)$ & $87(25.9)$ & \\
\hline $\mathrm{T} 4$ & 190(16.6) & $35(10.4 \square$ & \\
\hline Lymph node status & & & 0.001 \\
\hline Negative & $314(27.4)$ & $123(36.6)$ & \\
\hline Positive & $833(72.6)$ & $213(63.4)$ & \\
\hline Distant metastasis & & & 0.000 \\
\hline Mutiple site & $174(15.2)$ & $19(5.7)$ & \\
\hline Bone only & $98(8.5)$ & $9(2.7)$ & \\
\hline Brain only & $10(0.9)$ & $0(0)$ & \\
\hline Liver only & $56(4.9)$ & $11(3.3)$ & \\
\hline Lung only & $239(20.8)$ & $35(10.4)$ & \\
\hline Other site or distant LN only & $570(49.7)$ & $262(78.0)$ & \\
\hline Distant surgery & & & 0.000 \\
\hline No & 1070(93.3) & 247(73.5) & \\
\hline Yes & 77(6.7) & 89(26.5) & \\
\hline Chemotherapy & & & 0.003 \\
\hline No/unknown & $314(27.4)$ & 65(19.3) & \\
\hline Yes & $833(72.6)$ & $271(80.7)$ & \\
\hline Radiation & & & 0.001 \\
\hline No & $332(28.9)$ & $129(38.4)$ & \\
\hline Yes & $815(71.1)$ & 207(61.6) & \\
\hline Survival months & $10(4-20)$ & $22(11-36)$ & 0.000 \\
\hline Vital status & & & 0.000 \\
\hline Alive & $243(21.2)$ & $155(46.1)$ & \\
\hline Dead for this cancer & $782(68.2)$ & $151(44.9)$ & \\
\hline Dead for other cause & $109(9.5)$ & $25(7.4)$ & \\
\hline
\end{tabular}


livariate and multivariate analysis between clinicopathological characteristic and CCSS and OS of cervical cancer patients

\begin{tabular}{|c|c|c|c|c|c|c|c|c|}
\hline & \multicolumn{4}{|c|}{ CCSS } & \multicolumn{4}{|c|}{ OS } \\
\hline & \multicolumn{2}{|c|}{ Univariate analysis } & \multicolumn{2}{|c|}{$\begin{array}{c}\text { Multivariate } \\
\text { analysis }\end{array}$} & \multicolumn{2}{|c|}{ Univariate analysis } & \multicolumn{2}{|c|}{$\begin{array}{c}\text { Multivariate } \\
\text { analysis }\end{array}$} \\
\hline & HR(95\%CI) & $P$ & $\mathrm{HR}(95 \% \mathrm{CI})$ & $\mathrm{P}$ & $\mathrm{HR}(95 \% \mathrm{CI})$ & $\mathrm{P}$ & HR(95\%CI) & $\mathrm{P}$ \\
\hline 0 & Reference & & Reference & & Reference & & - & - \\
\hline 30 & $\begin{array}{l}1.01(0.84- \\
1.22)\end{array}$ & 0.905 & $\begin{array}{c}0.81(0.67- \\
0.98)\end{array}$ & 0.029 & $\begin{array}{c}1.10(0.92- \\
1.31)\end{array}$ & 0.315 & - & - \\
\hline 30 & $\begin{array}{c}1.14(0.93- \\
1.39)\end{array}$ & 0.213 & $\begin{array}{c}0.79(0.65- \\
0.97)\end{array}$ & 0.027 & $\begin{array}{c}1.31(1.09- \\
1.59)\end{array}$ & 0.005 & - & - \\
\hline 1 & $\begin{array}{l}2.07(1.48- \\
2.90)\end{array}$ & 0.000 & $\begin{array}{c}1.05(0.74- \\
1.48)\end{array}$ & 0.802 & $\begin{array}{c}2.59(1.91- \\
3.50)\end{array}$ & 0.000 & - & - \\
\hline te & Reference & & Reference & & Reference & & Reference & \\
\hline $\mathrm{k}$ & $\begin{array}{c}1.37(1.15- \\
1.62)\end{array}$ & 0.000 & $\begin{array}{c}1.34(1.12- \\
1.59)\end{array}$ & 0.001 & $\begin{array}{c}1.36(1.17- \\
1.60)\end{array}$ & 0.000 & $\begin{array}{c}1.31(1.12- \\
1.53)\end{array}$ & 0.001 \\
\hline irs & $\begin{array}{c}0.94(0.91- \\
1.40)\end{array}$ & 0.286 & $\begin{array}{c}1.07(0.86- \\
1.34)\end{array}$ & 0.554 & $\begin{array}{c}1.10(0.89- \\
1.35)\end{array}$ & 0.387 & $\begin{array}{c}1.02(0.83- \\
1.25)\end{array}$ & 0.879 \\
\hline \multicolumn{9}{|l|}{ type } \\
\hline $\mathrm{C}$ & Reference & & - & - & Reference & & - & - \\
\hline $\mathrm{C}$ & $\begin{array}{c}0.87(0.75- \\
1.02)\end{array}$ & 0.088 & - & - & $\begin{array}{c}0.90(0.78- \\
1.04)\end{array}$ & 0.143 & - & - \\
\hline \multirow[t]{7}{*}{ irs } & $\begin{array}{c}1.08(0.89- \\
1.32)\end{array}$ & 0.444 & - & - & $\begin{array}{c}1.08(0.90- \\
1.30)\end{array}$ & 0.426 & - & - \\
\hline & Reference & & - & - & Reference & & - & - \\
\hline & $\begin{array}{c}1.20(0.85- \\
1.70)\end{array}$ & 0.296 & - & - & $\begin{array}{c}1.18(0.86- \\
1.62)\end{array}$ & 0.302 & - & - \\
\hline & $\begin{array}{c}1.55(1.10- \\
2.16)\end{array}$ & 0.012 & - & - & $\begin{array}{c}1.48(1.09- \\
2.02)\end{array}$ & 0.012 & - & - \\
\hline & $\begin{array}{l}1.64(1.08- \\
2.49)\end{array}$ & 0.020 & - & - & $\begin{array}{c}1.71(1.17- \\
2.50)\end{array}$ & 0.005 & - & - \\
\hline & Reference & & Reference & & Reference & & Reference & \\
\hline & $\begin{array}{c}1.45(1.17- \\
1.80)\end{array}$ & 0.001 & $\begin{array}{c}1.34(1.08- \\
1.68)\end{array}$ & 0.009 & $\begin{array}{c}1.39(1.14- \\
1.69)\end{array}$ & 0.001 & $\begin{array}{c}1.33(1.09- \\
1.62)\end{array}$ & 0.006 \\
\hline i & $\begin{array}{c}2.11(1.73- \\
2.56)\end{array}$ & 0.000 & $\begin{array}{l}1.92(1.57- \\
2.35)\end{array}$ & 0.000 & $\begin{array}{c}1.94(1.63- \\
2.33)\end{array}$ & 0.000 & $\begin{array}{c}1.78(1.48- \\
2.14)\end{array}$ & 0.000 \\
\hline 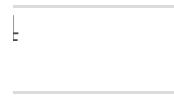 & $\begin{array}{l}2.15(1.70- \\
2.72)\end{array}$ & 0.000 & $\begin{array}{c}1.64(1.29- \\
2.08)\end{array}$ & 0.000 & $\begin{array}{l}2.06(1.67- \\
2.55)\end{array}$ & 0.000 & $\begin{array}{c}1.54(1.24- \\
1.492)\end{array}$ & 0.000 \\
\hline status & $\begin{array}{c}1.14(0.99- \\
1.31)\end{array}$ & 0.077 & $\begin{array}{c}1.20(1.04- \\
1.39)\end{array}$ & 0.015 & $\begin{array}{c}1.07(0.94- \\
1.22)\end{array}$ & 0.308 & - & - \\
\hline surgery & $\begin{array}{c}0.43(0.36- \\
0.51)\end{array}$ & 0.000 & $\begin{array}{c}0.67(0.53- \\
0.84)\end{array}$ & 0.001 & $\begin{array}{c}0.44(0.38- \\
0.52)\end{array}$ & 0.000 & $\begin{array}{c}0.69(0.56- \\
0.85)\end{array}$ & 0.000 \\
\hline I surgery & $\begin{array}{c}0.41(0.34- \\
0.50)\end{array}$ & 0.000 & $\begin{array}{c}0.68(0.53- \\
0.84)\end{array}$ & 0.002 & $\begin{array}{c}0.40(0.33- \\
0.48)\end{array}$ & 0.000 & $\begin{array}{c}0.63(0.50- \\
0.79)\end{array}$ & 0.000 \\
\hline ery & $\begin{array}{c}0.60(0.48- \\
0.75)\end{array}$ & 0.000 & - & - & $\begin{array}{c}0.62(0.51- \\
0.76)\end{array}$ & 0.000 & - & - \\
\hline \multirow[t]{2}{*}{ py } & $\begin{array}{c}0.37(0.32- \\
0.43)\end{array}$ & 0.000 & $\begin{array}{c}0.37(0.32- \\
0.43)\end{array}$ & 0.000 & $\begin{array}{c}0.36(0.31- \\
0.41)\end{array}$ & 0.000 & $\begin{array}{c}0.36(0.32- \\
0.42)\end{array}$ & 0.000 \\
\hline & $\begin{array}{c}0.66(0.57- \\
0.75)\end{array}$ & 0.000 & $\begin{array}{c}0.71(0.61- \\
0.81)\end{array}$ & 0.000 & $\begin{array}{c}0.62(0.54- \\
0.70)\end{array}$ & 0.000 & $\begin{array}{c}0.66(0.58- \\
0.76)\end{array}$ & 0.000 \\
\hline
\end{tabular}

istasis

: sites

Reference

Reference

Reference

Reference 


\begin{tabular}{lcccccccc} 
only & $0.54(0.41-$ & 0.000 & $\begin{array}{c}0.55(0.42- \\
0.73)\end{array}$ & 0.000 & $0.59(0.46-$ & 0.000 & $0.60(0.47-$ & 0.000 \\
& $0.71)$ & & $0.73)$ & $0.76)$ & & $0.78)$ & \\
\hline only & $0.77(0.38-$ & 0.462 & $0.66(0.32-$ & 0.259 & $0.87(0.46-$ & 0.668 & $0.65(0.35-$ & 0.194 \\
& $1.56)$ & & $1.36)$ & & $1.65)$ & & $1.24)$ & \\
\hline only & $0.62(0.45-$ & 0.003 & $0.65(0.47-$ & 0.008 & $0.61(0.45-$ & 0.001 & $0.59(0.44-$ & 0.001 \\
& $0.86)$ & & $0.90)$ & & $0.83)$ & & $0.80)$ & \\
\hline snLy & $0.56(0.45-$ & 0.000 & $0.51(0.41-$ & 0.000 & $0.60(0.49-$ & 0.000 & $0.52(0.43-$ & 0.000 \\
& $0.69)$ & & $0.63)$ & & $0.73)$ & & $0.64)$ & \\
\hline Jr distant & $0.30(0.25-$ & 0.000 & $0.34(0.29-$ & 0.000 & $0.31(0.26-$ & 0.000 & $0.37(0.31-$ & 0.000 \\
nly & $0.36)$ & & $0.42)$ & & $0.37)$ & & $0.44)$ & \\
\hline
\end{tabular}

\section{Figures}


A

ALL

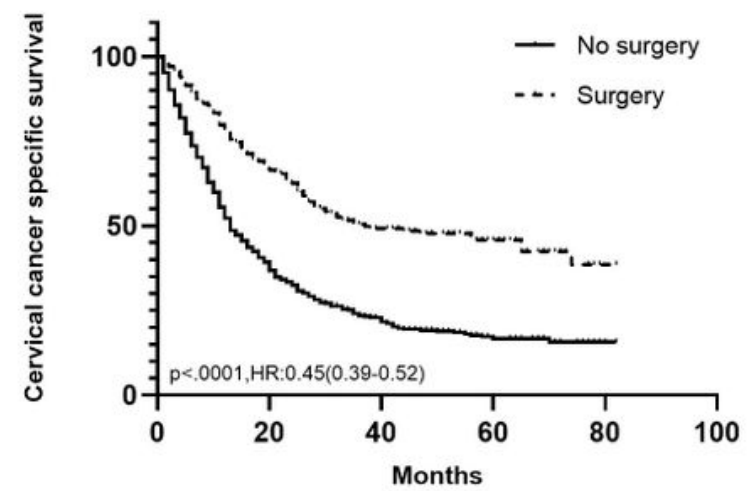

C

Bone only

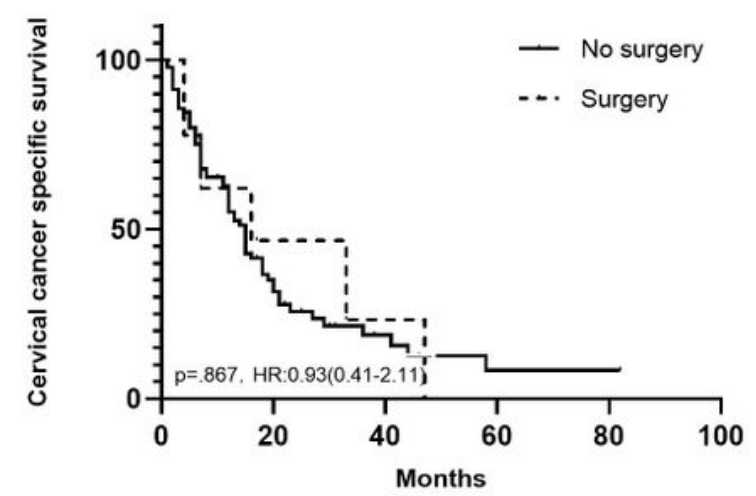

E

\section{Lung only}

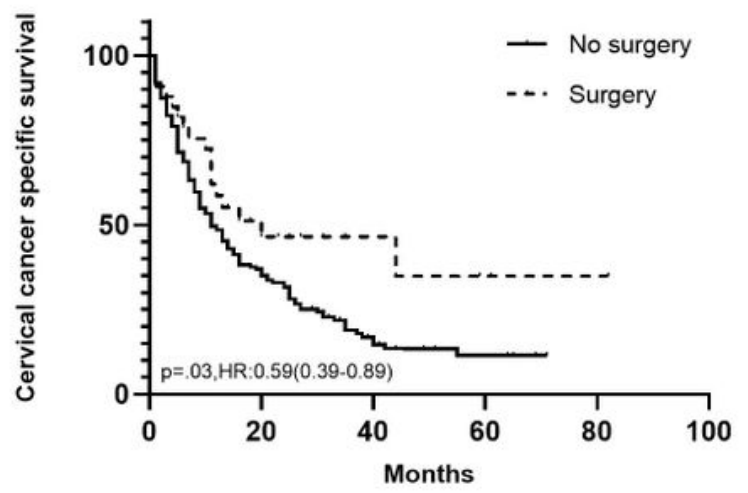

B

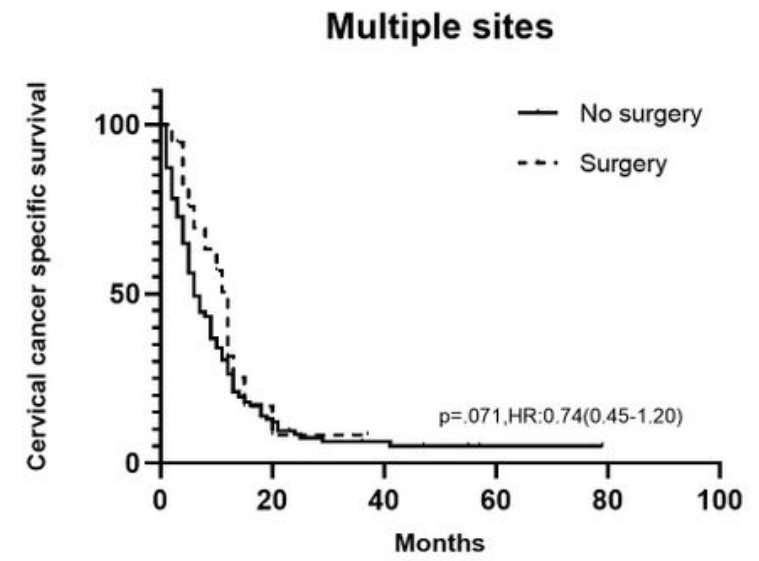

D

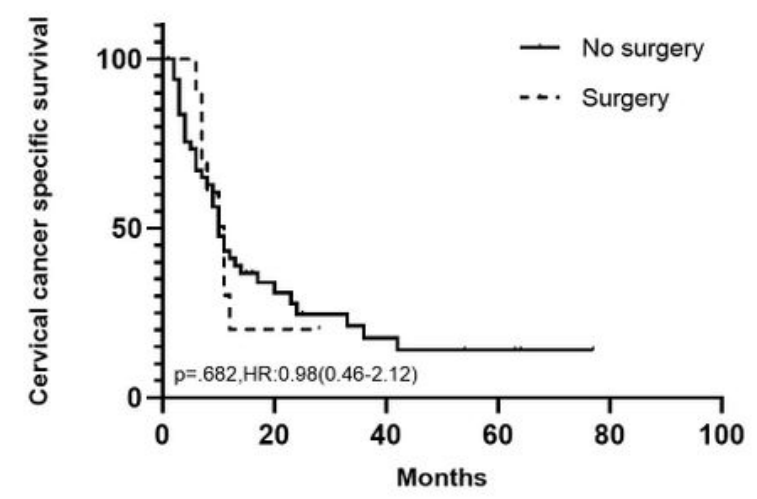

F

Other site or distant LN only

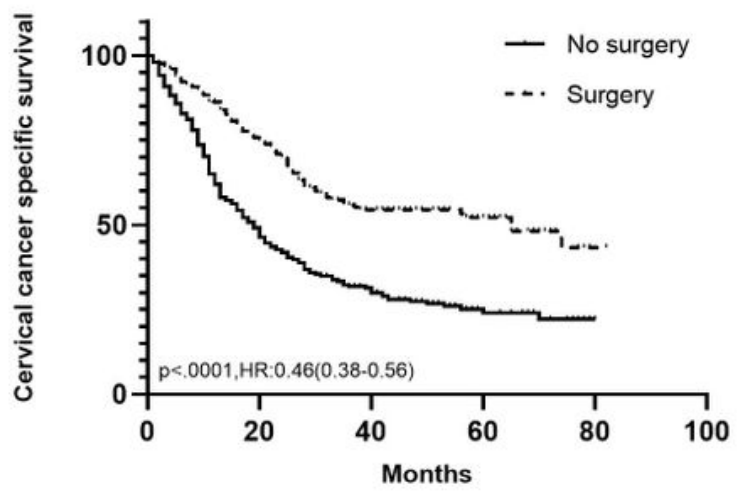

\section{Figure 1}

Cancer specific survival of FIGO IVB cervical cancer patients. Survival curve showing CCSS (A) in the surgery and no-surgery groups. B-F, CCSS curves classified by distant involved organs. 
A

ALL

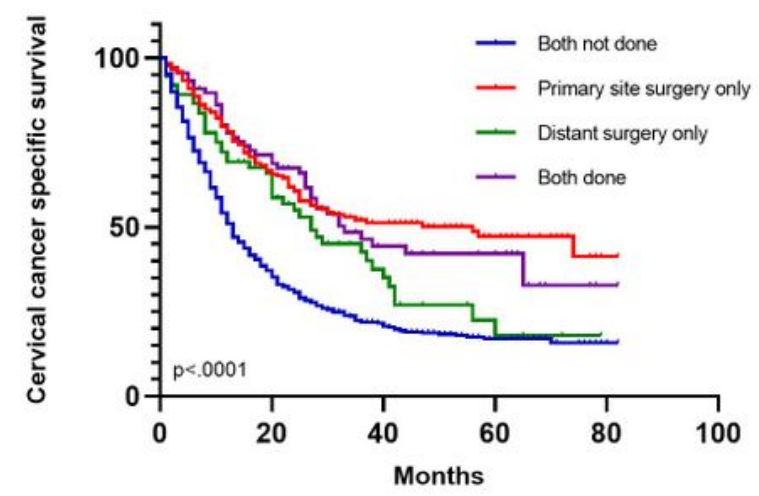

C

ALL

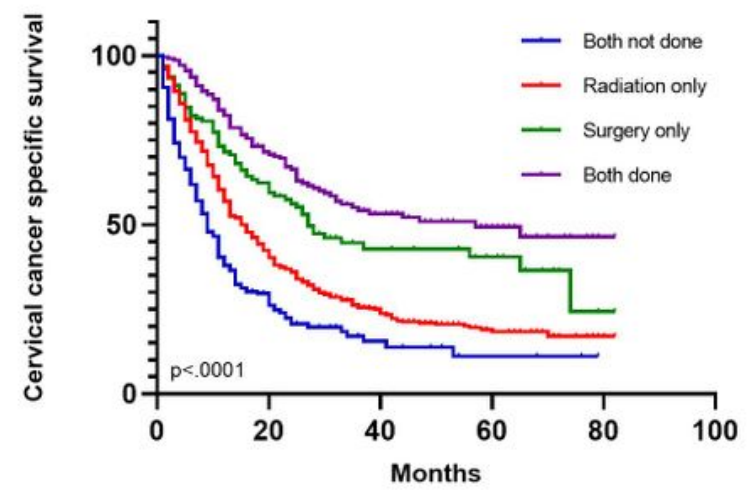

E

ALL

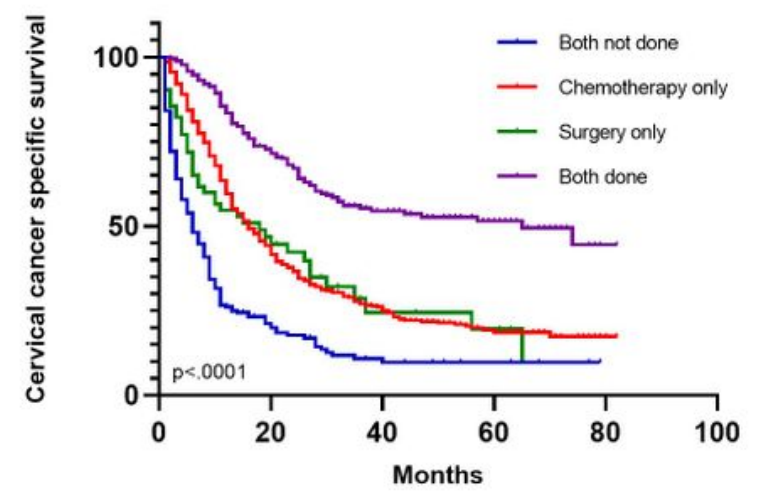

B

ALL

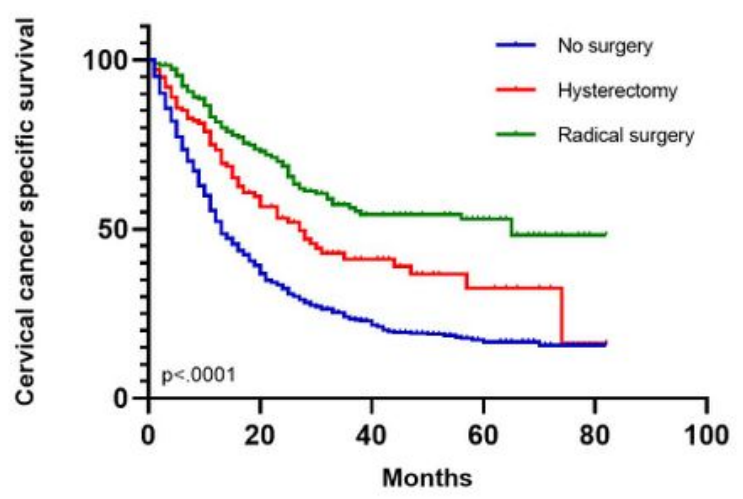

D

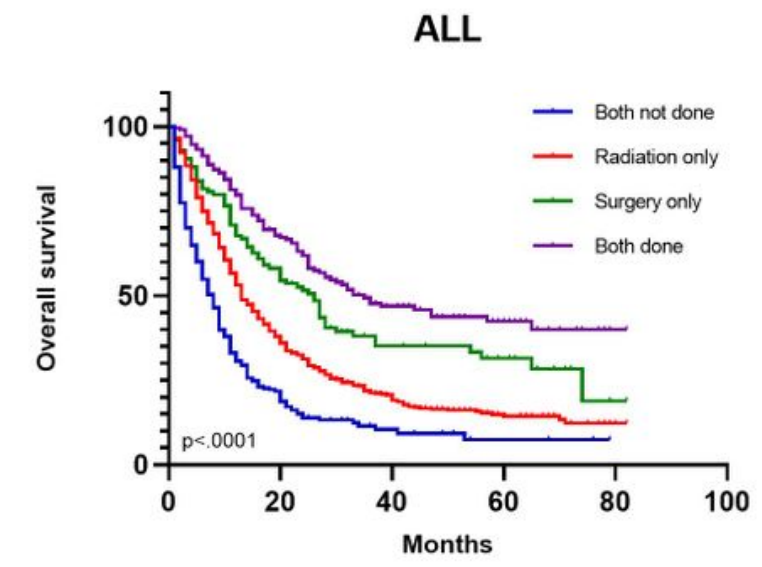

F

ALL

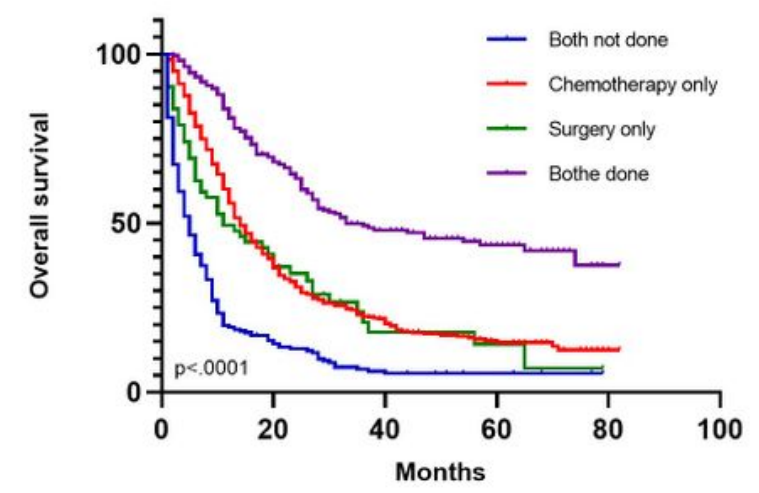

\section{Figure 2}

Survival of different treatment procedures. A, CCSS of FIGO IVB cervical cancer patients combined with primary site surgery and removal of distant metastasis. B, CCSS of patients with no primary surgery and patients who underwent hysterectomy, or radical surgery. C-D, survival of FIGO IVB cervical cancer with surgery combined with radiation or chemotherapy. 
A

B

scc

ADC

C
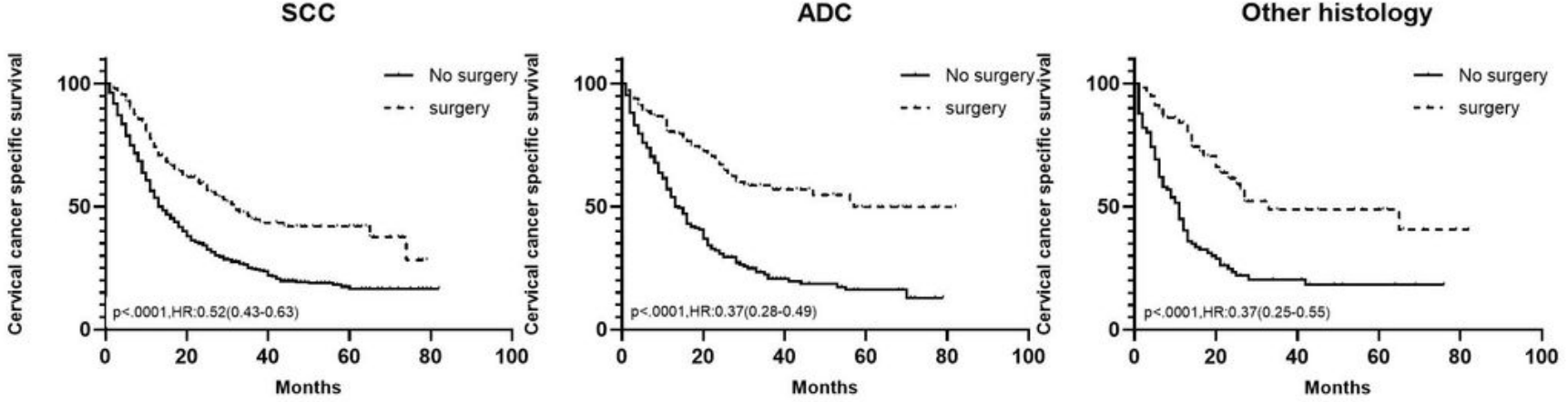

D

E

No

N+
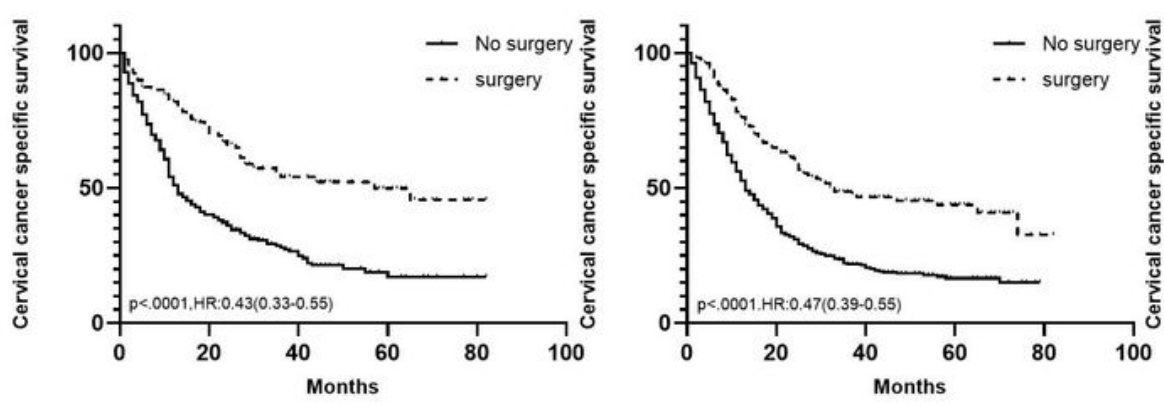

F

T1

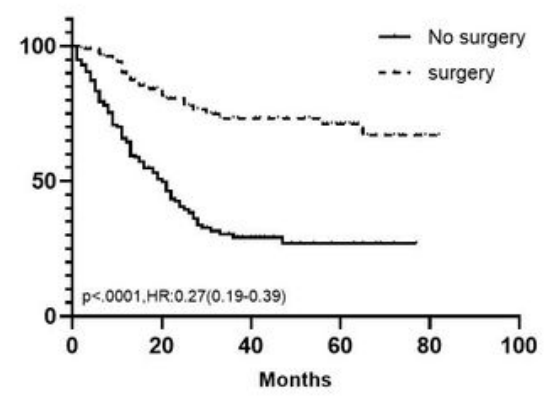

G

H

T3

T4
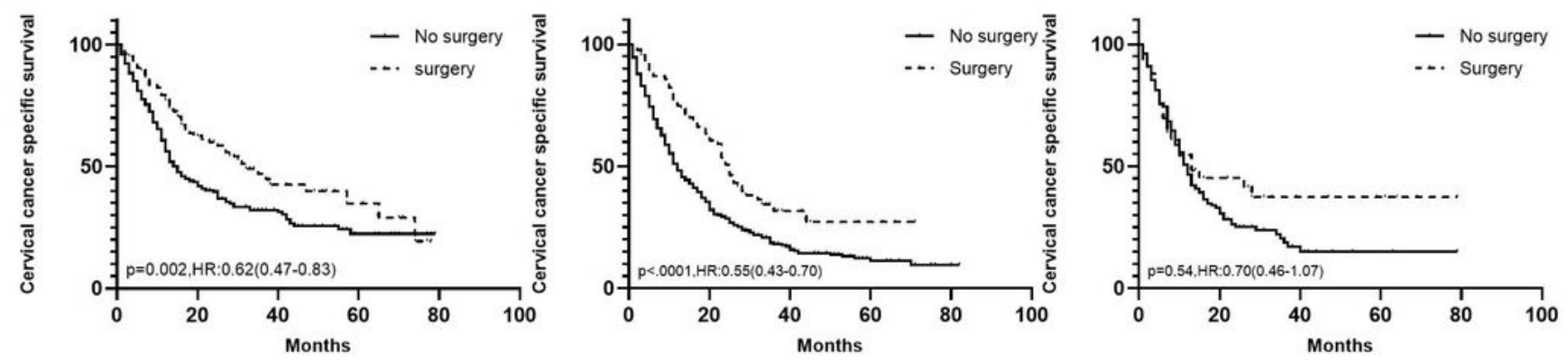

Figure 3

CCSS curves stratified by histological type, lymph node status and T stage.'

\section{Supplementary Files}

This is a list of supplementary files associated with this preprint. Click to download. 
- Additionalfile1.tif

Page 18/18 